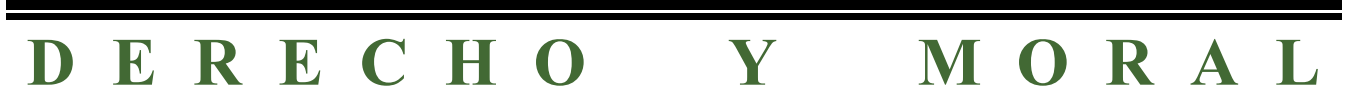




\section{DIMENSIONES MORALES DEL DERECHO}

I. DOS TIPOS DE JUICIOS MORALES: PRIMA FACIE Y «CONSIDERANDO TODAS LAS COSAS»

\section{Criterios de bondad moral}

xiste un considerable grado de consenso entre la gente, al menos en la cultura
occidental, acerca de que algunos principios son morales, y de que es prima facie
moralmente bueno guiarse por ellos.
Podríamos mencionar, por ejemplo, los siguientes principios (cfr. Peczenik 1989, 58 y sigs.):

1) No se debe prima facie causar daño a los demás.

2) Se debe prima facie ayudar a los demás.

3) Se debe prima facie trabajar con eficiencia.

4) Se debe prima facie decir la verdad.

5) Se debe prima facie mantener las propias promesas.

6) Se debe prima facie ser consecuente.

Además, juicios como los siguientes también están comúnmente aceptados en nuestra cultura:

1) A es prima facie una persona moralmente buena, ya que tiene una disposición a no causar daño a los demás.

2) A es prima facie una persona moralmente buena, ya que tiene una disposición a ayudar a los demás.

3) A es prima facie una persona moralmente buena, ya que tiene una disposición a trabajar con eficiencia.

4) A es prima facie una persona moralmente buena, ya que tiene una disposición a decir la verdad.

5) A es prima facie una persona moralmente buena, ya que tiene una disposición a mantener sus promesas.

6) A es prima facie una persona moralmente buena, ya que tiene una disposición a ser consecuente. 


\section{El carácter prima facie de los criterios morales establecidos}

Muchos criterios de bondad moral son significativos y además razonables. Yo creo, sin embargo, que tienen un carácter provisional, prima facie, por dos motivos (Sobre el concepto de «prima facie», cfr. Ross, 1930, 27-28).

En primer lugar, existe «la diversidad bien conocida entre los códigos morales de distintas sociedades y de distintos períodos históricos, y también las diferencias en las creencias morales entre diversos grupos y clases dentro de una misma comunidad compleja» (Mackie, 1977, 36).

Por otra parte, un mismo individuo a menudo abraza criterios que están en mutua competencia. Por ejemplo, un médico acepta simultáneamente la opinión de que debe informar a su paciente acerca de su enfermedad y la de que debe ayudarle con la mayor eficiencia posible. Pero la disposición del médico a decir la verdad puede en algunos casos perjudicar a su paciente. El médico debe entonces optar entre decir la verdad y ayudar eficientemente a su paciente.

También creo que la siguiente hipótesis es cierta: una elección racional de criterios y teorías de la bondad moral a menudo se basa en sopesar y equilibrar. Por ejemplo, un médico lleva a cabo un acto de sopesar, por el que decide si decir la verdad (un criterio moral) tiene en el presente caso un peso mayor o menor que el de evitar el daño (otro criterio).

Acciones lógicamente incompatibles pueden ser, al mismo tiempo, prima facie buenas. También podemos tener simultáneamente un deber prima facie de realizar acciones que son lógicamente incompatibles. La lógica «normal» no es por lo tanto aplicable a los juicios prima facie morales. Supongamos, por ejemplo, que A mató a B. Una razón prima facie -por ejemplo, las circunstancias de su acto- puede justificar una pena de cadena perpetua para A; otra -por ejemplo, la condición física de A-, puede apoyar un castigo más suave.

3. Dos conceptos de prima facie

Es necesario, sin embargo, trazar una distinción entre los siguientes conceptos de prima facie (cfr. Peczenik, 1989, 63 y sigs.).

1. Un juicio moral puede tener carácter prima facie-1, es decir, puede ser lingüísticamente posible. Tal es el caso si, y sólo si, el lenguaje en cuestión no hace que nos resulte extraño considerarlo dentro del acto de sopesar y equilibrar que determina nuestra opinión moral o conducta (acción u omisión). 
2. Un juicio moral puede tener también un carácter prima facie-2, es decir, puede ser relevante. Ello significa que la cultura en cuestión nos compele a tomarlo en consideración dentro del acto de sopesar y equilibrar que determina nuestra opinión moral o conducta (acción u omisión).

Sin intentar precisar seriamente la expresión vaga «la cultura en cuestión nos obliga a tomar en consideración un juicio normativo o valorativo», permítaseme ofrecer dos ejemplos de interpretaciones razonables de la misma:

a) Cualquier persona normal, perteneciente a esa cultura, en cualquier caso particular al que ese juicio es aplicable, vería como algo extraño no tomar en consideración tal juicio y sin embargo insistir en que se ha realizado un acto justificable de sopesar y equilibrar para responder a la pregunta de si $\mathrm{H}$ es definitivamente obligatorio o bueno.

b) Cualquier persona normal, perteneciente a esa cultura, en cualquier caso particular al que ese juicio es aplicable, actuaría de una manera que implica que está obedeciendo la regla según la cual se debe tomar en consideración tal juicio cuando se realice dicho acto de sopesar.

\section{La prueba relativa a las razones prima facie morales}

Nuestro conocimiento del contenido de los juicios morales que son relevantes en este sentido, viene respaldado por varios tipos de prueba.

Algunas veces tenemos acceso a textos y emisiones explícitos, según los cuales en contextos morales se debe tomar en consideración algunas razones. En otros casos, debemos confiar en una prueba implícita. Esta última consiste en otros textos, emisiones o acciones que apoyan una hipótesis acerca de una regla que obliga a considerar algunas otras razones morales. En otras palabras: tales textos, emisiones o acciones sólo son comprensibles si asumimos que la gente acepta implícitamente dicha regla.

La noción de prima facie implícita explica por qué es posible formular teorías innovadoras acerca del contenido de las razones prima facie posibles y relevantes. La gente que pertenece a la cultura en cuestión está continuamente realizando cada vez más acciones. Un teórico siempre puede crear nuevas hipótesis para explicar estas acciones.

\section{Principios y valores}

Algunos criterios de lo moralmente bueno se corresponden 
con algunos principios morales. Me ocuparé solamente del siguiente sentido de la palabra ambigua "principio»: un principio valorativo establece un ideal. Este ideal puede realizarse en un cierto grado, cuanto más alto mejor desde el punto de vista del principio (cfr. Alexy, 1985, 76).

La palabra «principio» también puede designar una norma general, una norma importante, etc. (Cfr. Alexy, 1985, 72 y sigs.; Dworkin, 1977, 14 y sigs.; Eckhoff, t980, 145 y sigs., con referencias a la literatura escandinava.)

Cada principio expresa un ideal; en otras palabras, un valor. Por ejemplo, un principio estipula que la igualdad, la libertad y la dignidad son valiosas. Un valor puede satisfacerse hasta un cierto grado, mayor o menor (cfr. Alexy, 1985, 130 y sigs.). Podemos expresar casi el mismo contenido con dos terminologías distintas, hablando de principios o hablando de valores. La única diferencia es la siguiente: un principio nos dice qué es prima facie obligatorio, mientras que un valor decide qué es prima facie lo mejor (Alexy, 1985, $133)$.

Cada principio, o valor, puede ser una razón prima facie para la acción. Pero los principios pueden colisionar de tal manera que, por ejemplo, un respeto incrementado a la igualdad, en el caso particular en cuestión, puede provocar una disminución de la libertad, y viceversa. Necesitamos entonces meta-razones («super-razones») para escoger entre ellos. En consecuencia, uno tiene meramente un deber provisional, prima facie, de seguir la literalidad de los principios. Los mismos tipos de valores y principios pueden aducirse en la «planta baja» del pensamiento moral, en su meta-nivel, en un meta-meta-nivel, etc.

La diferencia entre principios valorativos y reglas es más importante. Si alguien está en una situación regulada por una regla, sólo tiene dos posibilidades, obedecer la regla en cuestión o no obedecerla. La regla establece pues una frontera -precisa o vaga- entre lo obligatorio y lo no obligatorio, lo prohibido y lo permitido, etc. Si una acción o un estado de cosas se encuentra a la derecha de la frontera, la norma se obedece, por muy cerca que esté del límite. Una regla califica una acción humana como conforme o contraria a esa regla. Una propiedad importante de este modo de calificación es su carácter binario, 0 o 1 , todo o nada. Un principio valorativo, por el contrario, establece un ideal que puede realizarse en un cierto grado, mayor o menor. Califica una acción, una persona, etc., como más perfecta o me nos perfecta a la luz del principio. Un principio es una escala 
graduada para calificar. Este modo de calificación no es binario, sino por grados, de más a menos.

\section{Juicios prácticos «Considerando todas las cosas»}

Un juicio moral es definitivo sólo si, al emitirlo, uno declara que ya no está dispuesto a prestar atención a razones que justificarían la conclusión contraria.

Nuestra cultura exige que los juicios morales definitivos sean juicios morales «considerando todas las cosas». Tenemos un deber moral «considerando todas las cosas» de seguir el mejor compromiso, alcanzado después de sopesar y equilibrar diferentes principios valorativos (o juicios de valor).

Aquí es necesario hacer la siguiente distinción (cfr. Peczenik, 1989, 76 y sigs.):

Un juicio moral tiene la cualidad de «considerando todas las cosas» en sentido estricto si y sólo si está respaldado por consideraciones acerca de: a) todas las circunstancias moralmente relevantes, es decir, todos los hechos relevantes para el razonamiento práctico sobre ética, moral utilitarista, principios morales, derechos y deberes, virtudes, justicia, etc., y b) todos los criterios del razonamiento coherente. Ningún ser humano tiene recursos suficientes para formular juicios «considerando todas las cosas» en sentido estricto.

Nuestra cultura nos exige meramente aceptar juicios morales definitivos sólo si éstos tienen la cualidad de «considerando todas las cosas» en sentido amplio. Un juicio moral es «considerando todas las cosas» en sentido amplio si y sólo si está respaldado por consideraciones acerca de: a) tantas circunstancias moralmente relevantes como sea posible, y b) tantos criterios del razonamiento coherente como sea posible.

La expresión «tantos... como sea posible» indica aquí que ninguna consideración moral, y ningún criterio del razonamiento coherente, es por sí solo suficiente, sino que ha de ser contrapesado con otros criterios similares y con otros valores. Por ejemplo, en caso de emergencia, se debe salvar espontáneamente a una persona que se encuentre en peligro de muerte, en lugar de llevar a cabo un razonamiento moral que requiera un cierto tiempo.

Las acciones lógicamente incompatibles no pueden ser buenas «considerando todas las cosas» al mismo tiempo. Tampoco podemos tener simultáneamente un deber definitivo de realizar acciones lógicamente incompatibles. Si A, por ejemplo, debe de- 
finitivamente pagar a B cien coronas, es lógicamente imposible que A deba no hacerlo. La lógica es por lo tanto aplicable a los juicios morales «considerando todas las cosas».

\section{La tesis de la contextualidad}

Un típico juicio moral «considerando todas las cosas» se refiere a una situación individual: una persona determinada específicamente debe realizar una acción determinada específicamente, $\mathrm{H}$; o un objeto determinado específicamente es bueno, etc. La especificidad significa aquí que se consideran todas las circunstancias, todo el contexto de la situación.

Podemos optar ahora entre dos enfoques (cfr. Peczenik, 1989, 79 y sigs.):

1) Podemos suponer que cada situación es moralmente única, es decir, incluye al menos una circunstancia moralmente relevante no compartida por ninguna otra situación. Cada caso de evaluación moral es entonces «contextual» (cfr. Rentto, 1988, 64 y sigs.), o sea, único, caracterizado por un haz único de consideraciones que han de ser sopesadas. Podemos decir algo así: En la situación s-1, el valor v-1 realizado en la medida e-1 prevalece sobre el valor v-2 realizado en la medida e-2; y en la situación s-2 se da la relación inversa: el valor $\mathrm{v}-2$ realizado en la medida e- 2 prevalece sobre el valor $\mathrm{v}-1$ realizado en la medida e-l.

2) Podemos suponer que las situaciones individuales pueden ser clasificadas en tipos morales. Todas las situaciones que pertenecen a un mismo tipo se sopesan de la misma manera. Así, podemos decir en general que en la situación del tipo s-1, el valor v-1 realizado en la medida e-1 prevalece sobre el valor v-2 realizado en la medida v-2, etc... Bajo esta suposición, una regla general, o un juicio de valor general, puede tener un carácter «considerando todas las cosas» ceteris paribus, en el sentido siguiente: si las circunstancias permanecen inalteradas, es decir, nada nuevo y moralmente relevante acontece, entonces se debe seguir siempre la regla. O bien, si todas las circunstancias moralmente relevantes permanecen inalteradas, entonces un objeto de un cierto tipo es bueno, etc.

La opción entre estas suposiciones no es fácil. Pero incluso si se opta por la segunda, uno todavía podría sostener que absolutamente ninguna regla general puede ser, considerando todas las cosas, eternamente obligatoria. $\mathrm{Ni}$ tampoco un juicio de valor general puede ser, considerando todas las cosas, eternamente correcto. Uno podría, pues, sostener que el futuro siempre puede 
traer nuevas circunstancias que podrían adquirir relevancia moral.

Por esa razón, yo creo que ni siquiera los mejores cerebros filosóficos podrán jamás llegar a crear con éxito un cálculo que determine con precisión el contenido de la evaluación.

\section{El acto final de sopesar y equilibrar}

En cuanto sostenemos que un determinado principio pesa más que otro, nos enfrentamos inmediatamente con la pregunta de «¿Por qué?». Podemos apoyar la respuesta mediante razones ulteriores, inter alia principios. También éstos se pueden sopesar y equilibrar con otros posibles contraargumentos. Desde el punto de vista lógico, el proceso de sopesar puede así continuar ad infinitum. Pero en la práctica, tarde o temprano, uno debe poner fin a la argumentación.

Supongamos ahora, a efectos de la argumentación, que podemos formular la condición suficiente para llegar a la conclusión de que la acción en cuestión es, «considerando todas las cosas» (no sólo prima facie), buena y obligatoria. El hecho de que una razón moral, o una combinación de razones, es una condición suficiente para dicha conclusión, significa que ningún contraargumento concebible tiene más peso. Tal condición suficiente estaría formada por: 1) la lista completa de criterios y teorías morales prima facie, preestablecidas o de nueva creación, aplicables al caso en cuestión; y 2) la lista completa de juicios que determinan el peso relativo de dichos criterios y teorías en el presente caso.

La lista aludida puede estar compuesta por muchos niveles. En el nivel más bajo, hay razones a favor y en contra de la conclusión de que una determinada acción es buena y debe realizarse. En un nivel más alto, hay razones de segundo orden, a favor de una u otra forma de sopesar las razones y contraargumentos. Algunas razones de segundo orden establecen, por ejemplo, que ciertas razones de primer orden pesan más que sus correspondientes contraargumentos. La lista puede contener, por ejemplo, que ciertas razones de primer orden pesan más que sus correspondientes contraargumentos. La lista puede contener, por ejemplo, diez razones a favor y doce en contra de la conclusión de que debe realizarse una determinada acción, y una «super-razón» que establece que las diez pesan más que las doce. Estas «super-razones», a su vez, pueden sopesarse y equilibrarse 
frente a posibles contraargumentos. Puede haber, por tanto, razones de tercer orden, etc.

La lista es completa y no puede extenderse a ulteriores razones. Esta suposición se aplica a todos los niveles. No podemos añadir a la lista ninguna razón más, sea ésta de primer, segundo, o enésimo orden. Un razonamiento a favor de la conclusión de que las razones de la lista pesan más que los contraargumentos significa que uno simplemente repite algunas razones de un orden superior, que ya están en la lista. Para que las razones de la lista no constituyan un círculo lógico, la lista debe incluir una razón última, de orden «n», fundamental para toda la estructura argumentativa. Esta razón última tiene que ser aceptada sin ningún tipo de razonamiento (cfr. Peczenik, 1989, 82 y sigs.).

\section{arbitraria.}

De este modo, el último paso de un razonamiento es una presuposición

Este paso final de la evaluación puede consistir en un juicio concreto. Si la tesis de la contextualidad no es cierta, entonces el paso final en algunos casos puede consistir también en una regla general presupuesta, que determina un orden de prioridad entre los principios en cuestión. Por otra parte, es inconsistente decir que un principio constituye el paso final de la evaluación. Un principio, como ya dijimos, no es razón suficiente para una conclusión moral; ha de ser sopesado frente a otros principios. ¿Cómo debe ser pues el paso final de la evaluación?

\section{DERECHO PRIMA FACIE YMORALIDAD}

\section{El punto de partida: Interpretación valorativa en el Derecho}

Permítaseme pasar ahora a la relación entre moralidad y Derecho. El punto de partida es el siguiente. Todos los juristas conocen la importancia del papel que realmente juega la carga valorativa de las interpretaciones en la práctica del razonamiento jurídico. En pocas palabras, la interpretación jurídica es una actividad creativa y cargada de valor. «Interpretación», en el Derecho, no es una mera interpretación en sentido estricto, que establece el sentido lingüístico (léxico o contextual) de un texto jurídico. Incluye algo más, a saber, un perfeccionamiento del Derecho, su adaptación a la moral crítica. Dicho perfeccionamiento es una práctica común en los casos «difíciles». A la luz de esta práctica, el Derecho dado es meramente Derecho prima facie, y el Derecho perfeccionado es Derecho «considerando todas las cosas». 
2. La primera consecuencia: El carácter prima facie del Derecho socialmente establecido

La importancia que la interpretación cargada de valor tiene en el razonamiento jurídico hace que la siguiente tesis resulte plausible. El Derecho socialmente establecido, formulado mediante fuentes tales como leyes escritas, precedentes judiciales, trabajos preparatorios, etc., tiene un carácter prima facie. Las libertades, deberes, pretensiones, etc., formulados explícitamente por el Derecho socialmente establecido, son meramente prima facie jurídicas, ya que otras consideraciones pueden justificar la conclusión contraria en cuanto a que son deberes, pretensiones, etc., jurídicos (cfr. Peczenik, 1989, 239 y sigs.).

Por supuesto, los juristas y los abogados dan por sentadas algunas afirmaciones, que en su conjunto constituyen el paradigma jurídico o, dicho con otras palabras, el núcleo de la teoría jurídica. Este núcleo incluye, pues, algunos juicios morales fundamentales, comúnmente aceptados tanto entre los juristas como entre la gente que hace juicios morales. Incluye además el presupuesto de que el razonamiento jurídico está respaldado por el Derecho válido. También contiene ideas jurídicas fundamenta les acerca de la autoridad de las fuentes del Derecho y de las reglas del razonamiento jurídico. Por último, incluye algunas perspectivas valorativas fundamentales, sobre todo relativas a la seguridad jurídica y a la justicia (cfr. Aarnio, 1987, 17 y sigs.). Si queremos hacer un razonamiento jurídico, no podemos al mismo tiempo poner en cuestión una buena parte de este núcleo teórico. El contenido de estos presupuestos básicos del Derecho implica que no podemos dudar simultáneamente de gran parte del conjunto de normas, expresadas en leyes válidas, precedentes y otras fuentes básicas del Derecho. Sin embargo, podemos poner en duda cada presupuesto de este tipo y cada norma jurídica por separado. Pero la duda necesita justificación. Para justificar esta duda debemos contar con otras razones. En pocas palabras los presupuestos y normas jurídicas dadas tienen un carácter prima facie: constituyen razones prima facie, que han de ser sopesadas y equilibradas con otras razones.

Estas razones prima facie son de primer orden, para la realización de una determinada acción $\mathrm{H}$, y/o de segundo orden. Estas últimas requieren prima facie una exclusión de razones prima facie de primer orden, o sea, para hacer H. Considerando todas las cosas, una de estas razones de segundo orden puede justificar en algunos casos no hacer lo que se debería hacer en base al balance de las razones de primer orden. Por ejemplo, una 
disposición legal que prohíbe la inmigración puede justificar mi acción de no ayudar a los polacos menesterosos a establecerse en Suecia.

En el marco de la justificación jurídica contextualmente suficiente, es decir, dentro del paradigma jurídico, las razones jurídicas de ambos tipos son inmunes frente a algunas dudas. Una razón así es inmune frente a la pretensión de que su carácter de razón prima facie debería ser reexaminado de cara a una posible revisión en cada ocasión en que resulte aplicable. Por ejemplo, un abogado no puede dudar continuamente de la validez de cada disposición legal. Pero no es inmune frente a la pretensión de que tiene que dar prioridad a otras razones prima facie, si éstas son lo suficientemente poderosas. Ni tampoco es inmune frente a la pretensión de una posible revisión en el marco de una justificación profunda, fuera del paradigma jurídico.

Por cierto, esta idea es una paráfrasis de la teoría de las razones excluyentes en el Derecho, de Joseph Raz. No sé si Raz aceptaría esta paráfrasis. En cualquier caso, él ha sostenido lo siguiente. Una razón excluyente es una razón de segundo orden para no seguir una de primer orden. «Directamente», es una razón para excluir otra razón, R. para realizar una acción, H. «Indirectamente, debilita la postura a favor» de hacer $\mathrm{H}$. Una razón excluyente «nunca justifica abandonar nuestra autonomía, es decir, nuestro derecho y nuestro deber de actuar en base a nuestro propio juicio sobre qué es lo que se debe hacer, considerando todas las cosas». Pero puede justificar en algunos casos «no hacer lo que se debe hacer en base al balance de las razones de primer orden». Una razón excluyente «es inmune a la pretensión de que debería ser reexaminada de cara a una posible revisión en cada ocasión en que sea aplicable» (Raz, 1979, págs. 18, 27 y 33).

\section{Una objeción: El carácter no prima facie del Derecho establecido}

Por supuesto, un crítico puede admitir que la interpretación de las normas jurídicas puede llevar a un significado opuesto al significado literal y seguir negando el carácter prima facie de las normas. La razón es que el ordenamiento jurídico exige para sus derechos y deberes un status definitivo, y no simplemente un status prima facie.

El crítico puede plantear entonces las siguientes alternativas:

1. El Derecho establecido prevalece sobre la moral. El hecho de que otras consideraciones pueden justificar una conclusión contraria, implica la invalidez moral de dichas consideraciones, no el carácter prima facie del Derecho. 
2. El Derecho establecido es un sistema válido de normas, que puede ser incompatible con la moral válida. La «interpretación correctora» del Derecho establecido no es, desde esta perspectiva, un perfeccionamiento del Derecho, sino una creación de normas morales. Estas pueden ser o no moralmente válidas. Si son válidas, tienen un carácter «considerando todas las cosas» moral, pero pueden ser incompatibles con el Derecho «considerando todas las cosas».

Ambas versiones de la objeción implican una contradicción entre lo que el Derecho dice ser y lo que el Derecho debe ser a la vista de la práctica de su interpretación correctora (moral, cargada de valor). Para resolver esta contradicción, yo doy prioridad a la práctica. El crítico hace lo contrario; pero ¿por qué?

4. La segunda consecuencia: la obligación moral prima facie general de obedecer al Derecho.

Por otra parte, existe una obligación moral prima facie general de obedecer al Derecho (cfr. Peczenik, 1989, 242 y sigs.). Más concretamente:

Si el Derecho prima facie explícitamente contiene, implica o de cualquier forma apoya la conclusión de que A tiene un determinado deber, pretensión, potestad o privilegio jurídico sobre algo, entonces A tiene un deber, pretensión, potestad o privilegio prima facie moral con el mismo contenido.

Esta es una tesis de inclusión acerca de la relación entre lo prima facie jurídico y moral: el Derecho prima facie es, pues, una parte de la moral prima facie.

Desde luego, podemos imaginar algunos contraejemplos. Supongamos, por ejemplo, que un Derecho nazi contiene explícitamente una disposición según la cual la policía tiene un deber jurídico de matar a todo aquel que sea judío. ¿Tiene entonces la policía también el deber prima facie moral de matar judíos? Paradójicamente, la respuesta correcta es que sí. El hecho mismo de que esta disposición profundamente inmoral pertenece al Derecho socialmente establecido la convierte, por definición, en una razón moral prima facie significativa, que es, por supuesto, fácil de derrotar mediante otras razones prima facie morales. Esto es así a menos que uno niegue que el «Derecho» nazi sea realmente un sistema jurídico. Por supuesto, alguien puede negarlo por razones morales, pero entonces la inmoralidad tiene 
que subyacer sistemáticamente a todo el ordenamiento, incluidas sus disposiciones técnicas de Derecho privado, etc. Una disposición inmoral, o una rama del ordenamiento sistemáticamente inmoral, no es bastante.

Pero el deber, etc., moral de obedecer al Derecho es meramente un deber, etc., prima facie. El paso a la conclusión relativa a un correspondiente deber, etc., moral «considerando todas las cosas», presupone al menos una premisa adicional, que exprese un acto de sopesar y equilibrar acerca de la fuente del Derecho en cuestión y otras consideraciones. Al introducir la institución del ordenamiento jurídico, la sociedad puede restringir, pero no eliminar totalmente, la necesidad de sopesar y equilibrar.

5. Algunas razones a favor de la obligación moral prima facie general de obedecer al Derecho

Podemos proponer la siguiente justificación de estas relaciones entre el Derecho y los deberes, pretensiones, etc., morales prima facie (cfr. Peczenik, 1989, 243 y sigs.):

1. El razonamiento moral es relativamente indeterminado, como consecuencia de su dependencia última de la intuición y la voluntad.

Por supuesto, la moral no es arbitraria. Ya he señalado que nuestra cultura nos compele a aceptar algunas razones morales prima facie. Sin embargo, la moral consiste, ante todo, en principios que uno debe sopesar y equilibrar entre sí. Distintas personas pueden estar de acuerdo acerca de cuáles son los principios relevantes para el problema moral en cuestión, y sin embargo, discrepar al sopesarlos y equilibrarlos. Un caos moralmente objetable podría darse en una sociedad moderna, si ésta ya no tuviera un ordenamiento jurídico, es decir, un sistema normativo que esté bien determinado y hecho público. Es, por tanto, moralmente mejor tener una sociedad que posea un ordenamiento jurídico que en algunos casos conduce a decisiones moralmente incorrectas, que forzar a los individuos a confiar en sus propios juicios morales en todos los casos.

2. Razones todavía más fuertes apoyan nuestro deber de obedecer al Derecho en una sociedad democrática. La autoridad de los Derechos democráticamente creados se apoya, inter alia, en el principio de la mayoría. Este principio es una aproximación de un cálculo de preferencias humanas, aproximándose él mismo a la idea de lo moralmente bueno. 


\section{Moralidad 1 y moralidad 2}

Podemos decir, de forma algo paradójica, que las razones morales exigen obedecer al Derecho, en lugar de obedecer solamente a la moral.

En este contexto, quizá podamos distinguir entre dos tipos de consideraciones morales, a los que llamaremos «moralidad 1»y «moralidad 2». La moralidad 1 contiene algunos principios generales, por ejemplo, «no se debe denunciar al propio vecino ante las autoridades». La moralidad 2 determina el compromiso entre estos principios y el Derecho. Puede apoyar por lo tanto la siguiente conclusión: «se puede en algunos casos denunciar al propio vecino ante las autoridades, ya que hay una ley que lo exige»; esta conclusión es correcta sólo si el valor de la obediencia al Derecho pesa más que el principio en cuestión.

Únicamente la moralidad 2, y no la moralidad 1, establece deberes y valores «considerando todas las cosas», no meramente prima facie. En la moralidad 1 no se tiene en cuenta el Derecho. Pero el Derecho es un factor moralmente relevante. ¿Cómo puede uno decir entonces que tomó en consideración todos los factores moralmente relevantes?

\section{Una objeción: la obligación prima facie de no obedecer leyes inmorales}

Un crítico puede objetar que sólo algunas, no todas, las disposiciones jurídicas crean deberes morales prima facie (cfr. Peczenik, 1989, 247 y sigs.). El crítico puede ofrecer el siguiente conjunto de ejemplos: 1) Tenemos un deber moral prima facie de obedecer, por ejemplo, una norma que prohíbe aparcar vehículos en una calle muy concurrida, ya que la violación de esta norma produciría siempre un caos circulatorio. 2) En algunos casos, pero no en todos, un conductor tiene un deber moral prima facie de obedecer una señal roja de stop. Una violación a menudo produciría un caos, pero no tendría efectos moralmente significativos en una carretera desierta. 3) No tenemos un deber moral prima facie de obedecer, por ejemplo, una norma jurídica que establece que determinados contratos deben celebrarse en forma escrita. Esta norma es «moralmente neutra». 4) Por último, tenemos un deber moral prima facie de desobedecer una norma nazi que prohíbe a los judíos casarse con los «arios». Esta norma es inmoral prima facie.

A diferencia de este crítico, yo he presupuesto un deber moral prima facie de seguir cualquier Derecho, independientemente 
de su contenido. El contenido tiene muchísima importancia, pero sólo por lo que se refiere a los deberes morales «considerando todas las cosas», no a los prima facie. Considerando todas las cosas, debemos no seguir algunas normas nazis, pero prima facie debemos seguirlas. Esta interpretación de la expresión vaga «un deber moral prima facie» tiene las siguientes consecuencias: Es necesario un acto de sopesar y equilibrar para determinar los deberes morales «considerando todas las cosas». También puede ser necesario determinar si un cierto orden normativo en su conjunto es Derecho válido o no. Así, no sería Derecho válido si es tan extremadamente inmoral que produce caos y no orden. Tampoco sería Derecho válido si el orden que crea es peor que el caos. Pero no es necesario un acto de sopesar para establecer un deber moral prima facie de seguir las disposiciones que ya han sido reconocidas como jurídicamente válidas. Una disposición de este tipo puede que efectivamente produzca un mal orden, o incluso un caos. Pero todavía tenemos un deber moral prima facie de seguirla, ya que pertenece a un sistema cuya totalidad, en su conjunto, produce orden, y este orden es mejor que el caos.

El crítico, por el contrario, tiene que realizar siempre dos actos de sopesar: el primero, para establecer si una norma jurídica es prima facie moralmente obligatoria; el segundo, para determinar si es moralmente obligatoria «considerando todas las cosas».

\section{Un sistema normativo extremadamente inmoral no es Derecho válido.}

Por lo tanto yo sostengo que una vez que una disposición es jurídicamente obligatoria, también ha de ser, prima facie, obedecida en sentido moral, independientemente de su contenido. El crítico puede encontrar esto extraño, e insistir en que la obligación moral prima facie de obedecer una norma depende siempre del contenido de dicha norma.

El crítico tiene razón, pero solamente en el nivel de la validez externa, del ordenamiento jurídico en su conjunto. Debemos admitir que la validez jurídica de un sistema normativo como un todo no es totalmente independiente de su contenido. Un sistema normativo extremadamente inmoral no es Derecho válido. 


\section{EL CONCEPTO DE «DERECHO VALIDO»}

\section{La validez externa del sistema jurídico: hechos que crean Derecho} válido».

Llegados a este punto, debo decir algo más acerca del concepto de «Derecho

Existe una lista preestablecida de criterios de validez externa que determinan el hecho de que un sistema normativo como un todo es un sistema de Derecho válido (prima facie) socialmente establecido.

Podemos afirmar por lo tanto que la siguiente tesis es una explicación plausible de una relación analítica:

Existe al menos una descripción consistente de hechos (que hacen Derecho), tal que lo siguiente es cierto: si se dan estos hechos, entonces el sistema normativo S es prima facie Derecho válido.

Más concretamente, podemos afirmar que los siguientes hechos, inter alia, constituyen tales criterios de Derecho (cfr. Peczenik, 1989, 284 y sigs., con referencias más detalladas).

1. Algunos hechos versan sobre el contenido de las normas.

H-1) Un sistema jurídico consta de varios niveles; una determinada norma es válida si fue creada conforme a una norma de nivel superior.

H-2) Un sistema jurídico incluye no sólo normas de conducta, sino también reglas constitutivas que nos permiten hablar de hechos institucionales, tales como contratos, promesas, matrimonio, ciudadanía, etc.

H-3) Un sistema jurídico incluye algunas normas que establecen lo siguiente: El Derecho es el sistema supremo de normas en la sociedad; tiene el derecho exclusivo de autorizar el ejercicio de la fuerza física en su territorio; tiene autoridad para regular cualquier tipo de conducta.

2. Otros hechos versan sobre varios tipos de acción.

$\mathrm{H}-4)$ Un sistema jurídico incluye ciertas normas intencionalmente creadas por un complejo de diversas acciones que conjuntamente constituyen el proceso legislativo.

H-5) Un sistema jurídico es eficaz en el sentido siguiente: las normas de conducta más importantes que pertenecen a él son siempre o casi siempre observadas en la práctica de la gente corriente, cuando realizan acciones de la vida diaria como comprar, pagar impuestos, casarse, etc.; otras normas de conducta incluidas en este sistema son ampliamente observadas de esta forma; la mayoría de ellas al menos no son violadas sistemáticamente. 
H-6) Un sistema jurídico es también eficaz en otro sentido. Algunas normas de conducta importantes que pertenecen a él son siempre o casi siempre observadas en la práctica de los funcionarios públicos, aplicándolas así para afectar a las acciones de otros. Algunos de los funcionarios, por ejemplo, jueces, fiscales, policías, funcionarios de prisiones, etc., participan en el ejercicio de la fuerza jurídicamente autorizada.

H-7) El Derecho a menudo se publica y se aplica abiertamente; también es con frecuencia interpretado por juristas y abogados profesionales, utilizando doctrinas y métodos establecidos y altamente sofisticados.

\section{Hechos que crean deberes como hechos que crean Derecho}

Supongamos ahora que el sistema jurídico en cuestión, por ejemplo, el sistema de Pol Pot, contiene muchísimas disposiciones extremadamente inmorales. Es extremadamente inmoral como promedio, «im grossen und ganzen» (cfr. Kriele, 1979, especialmente 177; Dreier, 1982, 41 y sigs.). Resulta plausible suponer que este sistema «jurídico» no es ni siquiera prima facie un Derecho válido. Es moralmente mejor para una sociedad permitir a un individuo decidir todos los casos según su propio juicio moral que establecer un ordenamiento normativo que lleva con demasiada frecuencia a decisiones moralmente incorrectas. «Lex iniustissima non est lex».

Esta tesis puede compararse con la «tradición central del iusnaturalismo», que «ha afirmado que los Derechos injustos no son Derecho»... «Lex iniusta non est lex... implica (i) que algún significado-contenido normativo tiene para alguna comunidad el status... de Derecho (ii) que ese Derecho es injusto.... y (iii) que la obediencia a ese Derecho... no está justificada» (Finnis, 1980, 364-365). Pero «eso es despachar demasiado fácilmente el problema de los malos Derechos... Debemos decir por lo tanto... que lex iniustissima non est lex» (Lucas, 1980, 123).

Dado que el proceso legislativo democrático no es perfecto, pueden dictarse leyes injustas no sólo en un Estado totalitario, sino también en una sociedad democrática. Podemos criticarlas, incluso si aprobamos el sistema jurídico como tal. Los sistemas jurídicos de países como Sudáfrica o Cuba merecen una crítica más amplia, pero uno debe reconocer su carácter de Derecho válido. Sólo la inmoralidad extrema de un sistema normativo en su conjunto apoya la conclusión de que el sistema no es Derecho válido. 
Los siguientes hechos, inter alia, constituyen razones en contra de considerar un sistema normativo como extremadamente inmoral (cfr. Peczenik, 1989, 287 y sigs.):

H-8) El sistema normativo en cuestión no es tal que su aplicación causa un sufrimiento extremo.

H-9) El sistema normativo en cuestión no es tal que su aplicación contradice en grado extremo preferencias importantes de un número significativo de personas.

H-10) El sistema normativo en cuestión no es tal que su aplicación contradice claramente los objetivos que caracterizan importantes prácticas sociales.

H-12) El sistema normativo en cuestión no es tal que su aplicación es extremadamente injusta, ya que contradice en grado extremo el principio «los seres semejantes deben ser tratados de manera semejante».

H-13) El sistema normativo en cuestión no es tal que su aplicación es extremadamente injusta, ya que contradice en grado extremo el principio de que los miembros más débiles de la sociedad deben ser protegidos.

H-14) El sistema normativo en cuestión es tal que su aplicación es extremadamente injusta, ya que contradice en grado extremo el principio de que los individuos pueden decidir acerca de los productos de su propio trabajo.

\section{El carácter valorativamente abierto del Derecho válido}

Sin embargo, puede haber razones para ampliar la lista de los criterios de Derecho. No podemos determinar con precisión y objetividad criterios para la validez jurídica «considerando todas las cosas». Sólo podemos dar algunas razones prima facie, ni suficientes ni necesarias, tanto a favor como en contra de una determinada opción por una combinación de criterios. Al sopesar y equilibrar esas razones, decidimos la selección final de los hechos que uno considera suficientes y/ o necesarios para la validez jurídica. Así se decide, por ejemplo, acerca del carácter e intensidad de las propiedades que un sistema normativo debe reunir para ser Derecho válido.

Este acto de sopesar decide por lo tanto qué grado de perfección ha de tener la estructura jerárquica (H-1) de un sistema jurídico que merezca ese nombre; qué alcance han de tener sus pretensiones de supremacía, plenitud y monopolio de la fuerza (H-3); qué grado de eficacia ha de poseer el sistema (H-5, H-6), etc. Ese mismo acto de sopesar y equilibrar decide cuánto sufrimiento puede causar un sistema normativo, hasta qué punto puede ser injusto, ete. (H-8 - H-14), antes de 
negarle su carácter de Derecho válido. Al realizar ese acto de sopesar y equilibrar, podemos, por ejemplo, «compensar» las deficiencias morales del sistema con su elevada eficacia. Se podría decir, por ejemplo, que el sistema hitleriano de 1942, claramente eficaz, era Derecho válido, a pesar de atrocidades tales como el exterminio de los judíos. En 1945, sin embargo, la eficacia del sistema disminuyo y su injusticia aumentó tanto que uno podría poner en duda su validez jurídica (cfr. Peczenik, 1989, 289 y sigs.).

\section{DERECHO Y MORAL «CONSIDERANDO TODAS LAS COSAS»}

\section{Derecho «considerando todas las cosas» como Derecho interpretado.}

A este respecto, podemos hablar también de deberes, pretensiones, etc., jurídicos «considerando todas las cosas». El Derecho «considerando todas las cosas» es el resultado de la interpretación del Derecho prima facie. Dicho con más precisión: Un juicio jurídico es «considerando todas las cosas» en sentido amplio si y sólo si está apoyado en consideraciones relativas a a) tantas circunstancias jurídicamente relevantes como sea posible, y b) tantos criterios del razonamiento coherente como sea posible (cfr. Peczenik, 1989, 250 y sigs.).

2. La relación entre las normas jurídicas «considerando todas las cosas» y las normas morales «considerando todas las cosas»

Podemos considerar ahora una tesis de inclusión acerca de la relación entre lo «considerando todas las cosas» jurídico y lo «considerando todas las cosas» moral (cfr. Peczenik, 1989, 252 y sigs.):

Si una persona, A, tiene un deber, libertad, pretensión, etc., «considerando todas las cosas» jurídico, en relación con una determinada acción, $\mathrm{H}$, entonces esa persona tiene también un deber, libertad, pretensión, etc., «considerando todas las cosas» moral con el mismo contenido.

El Derecho «considerando todas las cosas», es decir, el Derecho óptimamente interpretado, es por lo tanto una parte de la moral «considerando todas las cosas». 


\section{Inclusión, no identidad}

La inclusión del Derecho «considerando todas las cosas» dentro de la moral «considerando todas las cosas» no es, sin embargo, una cuestión de identidad, por las siguientes razones:

1. Si una persona no tiene un deber, libertad, pretensión, etc., «considerando todas las cosas» jurídico con un cierto contenido, todavía puede tener un deber, libertad, pretensión, etc., «considerando todas las cosas» moral con el mismo contenido.

2. Si una persona tiene un deber, pretensión, etc., «considerando todas las cosas» jurídico con un cierto contenido y, en consecuencia, un deber, pretensión, etc., «considerando todas las cosas» moral con el mismo contenido, la identidad se refiere sólo al contenido, no a las razones que deberían aducirse explícitamente para apoyarlo. El mismo contenido, pues, recibe un respaldo distinto en el marco del razonamiento jurídico, y un respaldo distinto en el marco de un razonamiento moral. Si el segundo es completo, debe incluir al primero. Por el contrario, algunas razones morales pueden omitirse en una argumentación jurídica explícita. Esto es así incluso si la argumentación es óptima. Una argumentación jurídica óptima no requiere un apoyo explícito de todas las razones moralmente relevantes, aunque ciertamente requiere un apoyo implícito de todas ellas.

Supongamos, por ejemplo, que el tribunal tiene un deber jurídico «considerando todas las cosas» de ignorar los contratos orales de compraventa de inmuebles. La principal razón jurídica de este deber es, por supuesto, que el Derecho impone la forma escrita para tales contratos. Ahora bien, uno puede apoyar este deber mediante razones morales sustantivas. Pero para justificar tal deber moral, con independencia del Derecho, uno debe adoptar una visión amplia de la sociedad en su conjunto, y especular así sobre las consecuencias inmorales de la inseguridad relativa a la propiedad inmobiliaria, que supuestamente resultarían del reconocimiento de dichos contratos orales, etc. Tales consideraciones sustantivas pueden ser también apropiadas en el razona miento jurídico, pero ni siquiera la justificación jurídica óptima

necesita contener tanto de ellas como una justificación moral libre.

\section{Las lagunas en el Derecho interpretado. Interpretación jurídica y Crítica moral.}

El Derecho (prima facie) socialmente establecido constituye 
razones morales prima facie. Tenemos un deber prima facie de seguir el Derecho establecido. Pero hay un límite. Este deber prima facie debe ser sopesado frente a otras razones prima facie morales. No tenemos un deber «considerando todas las cosas» de seguir una norma jurídica injusta.

Esta concepción del Derecho, la moral y la racionalidad, implica que un individuo debe adoptar una actitud crítica hacia el Derecho. El puede criticar una decisión particular, un cierto número de normas jurídicas, o el sistema jurídico en su conjunto.

Podemos realizar dicha crítica dentro del marco del razonamiento jurídico ( «de lege data») o fuera de él, adoptando entonces el llamado punto de vista «de política jurídica» ( «de lege ferenda»). El primero permite ya al jurista reducir la injusticia del Derecho. Una persona que aplica el Derecho establecido puede sopesar su contenido literal frente a otras razones morales prima facie. Pero cuando el Derecho inmoral es claro, un intérprete del Derecho no puede hacer gran cosa. Sopesar no lleva a ningún resultado. Sería imposible entonces formular una norma que pudiera satisfacer simultáneamente dos condiciones necesarias de la interpretación jurídica, a saber: 1) que tuviera un apoyo relevante de las normas jurídicas socialmente establecidas; y 2) que tuviera un apoyo suficiente de normas morales prima facie. En un caso así, simplemente no existe una norma jurídica «considerando todas las cosas». En cuanto prestamos atención al Derecho establecido, tenemos que dejar de lado la moral, y viceversa. Ninguna norma jurídica «considerando todas las cosas» puede basarse en la norma jurídica socialmente establecida en cuestión. En consecuencia, ninguna norma jurídica definitiva que uno adopte puede ser correcta. Hay una laguna en el Derecho; no ya simplemente en el Derecho prima facie, socialmente establecido, sino en el Derecho «considerando todas las cosas», interpretado (cfr. Peczenik, 1989, 254 y sigs.).

(Trad. de Juan A. Pérez Lledó) 


\section{BIBLIOGRAFÍA}

Aarnio, Aulis, 1987, The Rational as Reasonable, Dordrecht/ Boston/ Lancaster/ Tokio: Reidel.

Alexy, Robert, 1985, Theorie der Grundrechte, Baden-Baden: Nomos (Frankfurt/M. 1986: Suhrkamp).

Dworkin, Ronald, 1987, Taking Rights Seriously, Cambridge, Mass.: Harvard University Press.

Ekhoff, Torstein, 1980. Retningslinjer og «tumregler», Tidskrift for Rettsvitenskap. Finnis, John, 1980, Natural Law and Natural Rights, Oxford: Clarendon Press.

Kriele, Martin, 1979, Recht und Praktische Vernunft, Göttingen: Vandenhoek \& Ruprecht. Lucas, J. R., 1980, On Justice, Oxford, Clarendon Press.

Mackie, John L., 1977, Ethics. Inventing Right and Wrong, Harmondsworth: Penguin.

Peczenik, Aleksander, 1989, On Law and Reason, Dordrecht/ Boston/ London: Kluwer.

Raz, Joseph, 1979, The Authority of Law, Oxford, Clarendon Press.

Rentto, Juha-Pekka, Prudentia Juris. The Art of the Good and the Just, Turku: Turun Yliopisto.

Ross, W. D., 1930, The Right and the Good, Oxford, Clarendon Press. 